\title{
Entropy and Multifractality in Relativistic Ion-Ion Collisions
}

\author{
Shaista Khan and Shakeel Ahmad \\ Department of Physics, Aligarh Muslim University, Aligarh 202002, India \\ Correspondence should be addressed to Shakeel Ahmad; shakeel.ahmad@cern.ch
}

Received 31 August 2017; Revised 5 January 2018; Accepted 11 March 2018; Published 11 April 2018

Academic Editor: Raghunath Sahoo

Copyright (C) 2018 Shaista Khan and Shakeel Ahmad. This is an open access article distributed under the Creative Commons Attribution License, which permits unrestricted use, distribution, and reproduction in any medium, provided the original work is properly cited. The publication of this article was funded by SCOAP ${ }^{3}$.

\begin{abstract}
Entropy production in multiparticle systems is investigated by analyzing the experimental data on ion-ion collisions at AGS and SPS energies and comparing the findings with those reported earlier for hadron-hadron, hadron-nucleus, and nucleus-nucleus collisions. It is observed that the entropy produced in limited and full phase space, when normalized to maximum rapidity, exhibits a kind of scaling which is nicely supported by Monte Carlo model HIJING. Using Rényi's order $q$ information entropy, multifractal characteristics of particle production are examined in terms of generalized dimensions, $D_{q}$. Nearly the same values of multifractal specific heat, $c$, observed in hadronic and ion-ion collisions over a wide range of incident energies suggest that the quantity $c$ might be used as a universal characteristic of multiparticle production in hadron-hadron, hadron-nucleus, and nucleus-nucleus collisions. The analysis is extended to the study of spectrum of scaling indices. The findings reveal that Rényis order $q$ information entropy could be another way to investigate the fluctuations in multiplicity distributions in terms of spectral function $f(\alpha)$, which has been argued to be a convenient function for comparison sake not only among different experiments but also between the data and theoretical models.
\end{abstract}

\section{Introduction}

The main objective of investigating the collisions of heavy nuclei at relativistic energies is to understand the nature of phase transition from normal hadronic matter to quarkgluon plasma (QGP) [1]. Dedicated experiments have been carried out at AGS and RHIC at BNL and SPS and LHC at CERN to search for the QGP phase and explore the QCD phase diagram. Heavy-ion collision experiments provide a unique opportunity to test the predictions of QCD and at the same time to understand the soft processes involved, which dominate even at LHC energies, as well as the hard scattering or the small cross-sectional physics [2-4]. Relativistic nucleus-nucleus (AA) collisions serve as a mean to study the novel regime of QCD where parton densities are high enough while the strong coupling constant between the partons is small and decreases further with decreasing interpartonic distances [2]. The parton densities in the early stage of the collision can be related to the density of the produced charged hadrons in the final state. Dominance of hard process (jets and minijets) in the hadron production increases with increasing collision energy and hence provides a unique opportunity to study the interplay between effects. In this scenario, the perturbative QCD (pQCD) lends a good basis for high energy dynamics and has achieved significant success in describing the hard processes involved in high energy collisions [2, 5]. Hadronic-jet production in $e^{+} e^{-}$annihilations $[6,7]$ and large $p_{t}$ jet production in hadron-hadron $(\mathrm{hh})$ collisions $[8,9]$ are the examples of these hard processes. However, in soft processes like hadron production with sufficiently low $p_{t}$ in hadronic and heavyion collisions, the interactions become so strong that the pQCD is not applicable anymore [2]. Due to incapability of pQCD in this regime, phenomenological models based on experimental inputs have been proven to be an alternative tool to understand the dynamics of particle production in AA collisions.

Relativistic charged particle multiplicity is the simplest observable and by studying its distribution for a given data sample, information on soft QCD processes as well as on hard scattering can be extracted [3]. Furthermore, the charged hadron multiplicity of an event is taken as a direct measure of its inelasticity and can describe important features of particle production [3]. Investigations involving 
multiparticle production in $\mathrm{hh}, \mathrm{hA}$, and AA collisions at relativistic energies have been carried out by numerous groups during the last four decades [10-15]. However, a complete understanding of particle production mechanism still remains elusive. Multiplicity distributions (MD) of relativistic charged particles produced in hh collisions have been observed to deviate from a Poisson distribution and are expected to provide information regarding the underlying production mechanism $[13,16]$. Asymptotic scaling of MD in hh collisions referred to as the KNO scaling [17], predicted in 1972, was regarded as a useful phenomenological framework for comparing the $\mathrm{MD}$ at different energies ranging from $\sqrt{s} \sim 10 \mathrm{GeV}$ to ISR energies [18]. It was, however, pointed out $[19,20]$ that KNO scaling is not strictly followed for inelastic hh collisions. This scaling law was observed to breakdown when collision energy reached SPS range $[13,16$, $18,21]$. After the observations of KNO scaling violation in $\bar{p} p$ collisions at $\sqrt{s}=540 \mathrm{GeV}$, it was remarked that the observed scaling up to ISR energies was approximate and accidental [21]. A new empirical regularity, in place of KNO scaling, was then proposed [21] to predict the multiplicity distributions at different energies. It was shown that $\mathrm{MD}$ at different energies in full and limited rapidity $(\eta)$ windows may be nicely reproduced by negative binomial distributions (NBD) $[18,21,22]$. These observations lead to revival of interest in investigations involving $\mathrm{MD}$ and new scaling laws. Simak et al. [23], by introducing a new variable, the information entropy, showed that MD of charged particles produced in full and limited phase space in hh collisions exhibits a new type of scaling law in the energy range, $\sqrt{s} \sim 19$ to $900 \mathrm{GeV}$.

Sinyukov and Akkelin [24] have proposed a method to estimate entropy of thermal pions in AA collisions and have studied the average phase space densities and entropy of such pions against their multiplicities and beam energies. Their findings apparently suggest the presence of deconfinement and chiral phase transition in relativistic AA collisions. Moreover at RHIC energies, entropy per unit rapidity at freezeout has been extracted with minimal model dependence from the available measurements of particle yields, spectra, and source sizes, determined from two-particle interferometry [25]. The extracted entropy per unit rapidity was observed to be consistent with the lattice gauge theory for thermalized QGP with an energy density calculated from the transverse energy production at RHIC energies.

Analyses of the experimental data on $p p, \bar{p} p$, and $k^{+} p$ collisions over a wide energy range (up to $\sqrt{s}=900 \mathrm{GeV}$ ) carried out by several workers $[23,26,27]$ indicate that entropy increases with beam energy while the entropy per unit rapidity appears to be an energy independent quantity. These results indicate the presence of entropy scaling up to a few $\mathrm{TeV}$ energy. Presence of a similar scaling behaviour in $p p$ collisions at LHC energies has also been reported by Mizoguchi and Biyajima [28] and Das et al. [11, 13]. Analyses of AA collision data at AGS and SPS energies carried out by other workers $[10,15,29,30]$ too suggest that entropy produced in limited pseudorapidity $(\eta)$ windows when normalized to the maximum rapidity is, essentially, independent of projectile and target mass as well as the beam energy, indicating the presence of entropy scaling.
The occurrence of unusual large particle density fluctuations in narrow phase space bins, observed in cosmic ray JACEE events [31] and in accelerator experiments [32, 33], have generated considerable interest in the study of nonlinear phenomena in hadronic and heavy-ion collisions. Such fluctuations may be taken as an indication of a phase transition from ordinary hadronic matter to QGP, predicted by QCD to occur in relativistic AA collisions. These fluctuations are also envisaged to arise either due to minijets produced at very high energies or (and) because of some other collective phenomena [34]. Such rare fluctuations of dynamical origin are to be identified and extracted from the statistical ones. Various methods for the identification of these fluctuations have been proposed which estimate the total deviation of the measured rapidity distribution from an idealized smooth distribution [34]. The analysis is based on the estimation of corresponding statistical probability through Monte Carlo (MC) simulations. The method of scaled factorial moments (SFMs), proposed by Bialas and Peschanski [35], has been proven to be well suited for not only to search for the dynamical fluctuations in narrow phase space bins but also to investigate the pattern of fluctuations which could lead to a physical interpretation of their origin [35]. The concept of SFMs was put forward in analogy with the phenomenon referred to as the "intermittency" in hydrodynamics of turbulent fluid flow [34]. This phenomenon is characterized by the presence of fluctuations in a small part of the available phase space. The method of SFMs was first applied to the pseudorapidity distribution of a single high multiplicity JACEE event [31] and the findings indicated the presence of intermittent pattern (large particle density fluctuations in narrow pseudorapidity bins) [35]. SFMs analysis, since its introduction, has been widely used to search for the nonlinear phenomena in hadronic and ion-ion collisions. It has been observed [36-39] that the presence of such a nonlinear phenomena might be rare but not impossible [40]. Such fluctuations, if not arising due to the statistical reasons, are envisaged to occur due to the dynamical correlations among the produced particles. These correlations might arise due to the phase transition from QGP to normal hadronic matter. As pointed out by Bialas and Peschanski [35], if the intermittency exists, the SFMs of multiplicity distributions should exhibit a power law dependence of the rapidity-bin width, as $\delta y \rightarrow 0$. Such a study would help understand the chaotic behaviour of rapidity distribution on event-by-event (ebe) basis instead of investigating the average phenomena [41].

The observations of intermittency in $e^{+} e^{-}$annihilation [42], hh [43], hA [34], and AA collisions [41] attracted a great deal of attention towards the investigations involving the power law behaviour of SFMs of the form

$$
F_{q} \propto(\delta y)^{\alpha(q)}, \quad \delta y \longrightarrow 0,
$$

where the exponent $\alpha(q)$ increases with order $q$ of the moment [41].

Although the intermittency analysis in terms of SFMs has been successfully applied to the hadronic and heavyion collisions, yet the dynamical explanation of its origin in 
some cases is not clear [44]. The concept of self-similarity is closely related to the fractal theory $[45,46]$ which is a natural consequence of the cascading mechanism prevailing in the multiparticle production. A formalism for treating the fractal dimensions and its generalization has been developed and applied to the study of turbulent fluids and other transitions to chaos [41, 47]. However, in the case of multiparticle production, the dynamics is not well known and one has to discover the proper dynamics that gives rise to the chaotic structure. The fractal dimensions, in multiparticle production, were first studied by Carruthers and DuongVan [48]. Later on Dermin [49] suggested the study of correlation dimensions, while Lipa and Buschbeck [50] considered other generalized dimensions. However, in none of these investigations a formalism for systematic studies of fractal properties has been presented. Considering inelastic collisions as purely geometrical objects with noninteger dimensions, a formalism for treating the fractal dimensions and its generalization was developed and successfully applied to the study of intermittent behaviour and other transitions to chaos [44, 47, 51-53]. In order to reveal the multifractal nature of multiparticle production, Hwa [41], Chiu and Hwa [54], and Florkowski and Hwa [55] introduced $G_{q}$ moments, which can be estimated from the particle multiplicities in limited rapidity windows that might lead to inferences on the nature of the dimensions, $D_{q}$, that are the generalizations of the fractal dimensions for multifractal sets. Using the $G_{q}$ moments properties of scaling indices spectrum may be investigated which, in turn, would indicate as to how it can provide effective means to describe a highly nonuniform rapidity distribution.

As the studies involving AA collisions at relativistic energies are concerned, the main goal is to study the properties of strongly interacting matter under extreme conditions of nuclear density and temperature, where formation of quarkgluon plasma (QGP) is predicted to take place $[10,15,56,57]$. Fluctuations in physical observables in AA collisions are regarded as one of the important signals, for QGP formation because of the idea that, in many body systems, phase transition may result in significant changes in the quantum fluctuations of an observable from its average behaviour [15, $29,56]$. For example, when a system undergoes a phasetransition, heat capacity changes abruptly, whereas the energy density remains the smooth function of temperature [10, $15,25,58,59]$. Entropy is regarded yet another important characteristics of a system with many degrees of freedom $[15,60-63]$. Processes in which particles are produced may be considered as the so-called dynamical systems [60-64] in which entropy is generally produced. Systematic measurements of local entropy produced in AA collisions may provide direct information about the internal degrees of freedom of the QGP medium and its evolution $[10,15,27]$. It has been pointed out [65] that in high energy collisions particle production occurs on the maximum stochasticity; that is, they should follow the maximum entropy principle. This type of stochasticity may also be quantified in terms of information entropy which may be regarded as a natural and more general parameter to measure the chaoticity in branching processes [66].
TABLE 1: Number of events selected for the analysis.

\begin{tabular}{lcc}
\hline Energy $(\mathrm{GeV} / \mathrm{c})$ & Type of interactions & Number of events \\
\hline $10.6 \mathrm{~A}$ & ${ }^{197} \mathrm{Au}-\mathrm{AgBr}$ & 577 \\
$14.5 \mathrm{~A}$ & ${ }^{16} \mathrm{O}-\mathrm{AgBr}$ & 379 \\
$14.5 \mathrm{~A}$ & ${ }^{28} \mathrm{Si}-\mathrm{AgBr}$ & 561 \\
$60 \mathrm{~A}$ & ${ }^{16} \mathrm{O}-\mathrm{AgBr}$ & 422 \\
$200 \mathrm{~A}$ & ${ }^{16} \mathrm{O}-\mathrm{AgBr}$ & 223 \\
$200 \mathrm{~A}$ & ${ }^{32} \mathrm{~S}-\mathrm{AgBr}$ & 452 \\
\hline
\end{tabular}

Bialas et al. [60, 61, 67] proposed that Rényi entropies may also be used as a tool for studying the dynamical systems and are closely related to the thermodynamic entropy of the system, the Shannon entropy. Bialas et al. [68] have also suggested that Rényi's entropies may serve as a useful tool for examining the correlation among the particles produced in high energy collisions. Furthermore, the generalization of Rényi's order- $q$ information entropy contains information on the multiplicity moments that can be used to investigate the multifractal characteristics of particle production $[14,69,70]$. It should be mentioned that this method of multifractal studies is not related to the phase space binwidth or the detector resolution but to the collision energy [69]. It is, therefore, considered worthwhile to carry out a well focused study of entropy production and subsequent scaling in AA collisions by analyzing the several sets of experimental data on AA collisions at AGS and SPS energies. Rényi's information entropies are also estimated to investigate the multifractal characteristics of multiparticle production. Moreover, the analysis is further extended to study the fractal dimensions, multifractal spectrum, and their dependence on the energy and mass of the projectile.

\section{Details of Data}

Six sets of events produced in collisions of ${ }^{16} \mathrm{O},{ }^{28} \mathrm{Si}$, ${ }^{32} \mathrm{~S}$, and ${ }^{197} \mathrm{Au}$ beams with $\mathrm{AgBr}$ group of nuclei in emulsion at AGS and SPS energies, available in the laboratory, are used in the present study. Details of these samples are presented in Table 1. These events are taken from the emulsion experiments performed by EMU01 Collaboration [71-74]. The other relevant details of the data, like selection criteria of events, classification of tracks, extraction of $\mathrm{AgBr}$ group of interactions, method of measuring the emission angle, $\theta$ of relativistic charged particles, and so on, may be found elsewhere [10, 15, 71, 75-77].

From the measured values of the emission angle $\theta$, the pseudorapidity variable, $\eta$, was calculated using the relation, $\eta=-\ln \tan (\theta / 2)$. It is worthwhile to mention that the conventional emulsion technique has two main advantages over the other detectors: (i) its $4 \pi$ solid angle coverage and (ii) emulsion data being free from biases due to full phase space acceptance. In the case of other detectors, only a fraction of charged particles is recorded due to the limited acceptance cone. This not only reduces the multiplicity but also may distort some of the event characteristics like particle density fluctuations $[15,75,78]$. In order to compare the findings of the present work with the predictions of Monte Carlo 
model HIJING $[79,80]$, event samples corresponding to the experimental ones are simulated using the code HIJING1.35; the number of events in each simulated sample is equal to that in the corresponding real event sample. The events are simulated by taking into account the percentage of interactions which occur in the collision of projectile with various targets in emulsion which constitute the $\mathrm{AgBr}$ group of nuclei $[71,74,75]$. The value of impact parameter for each data sample was so set that the mean multiplicity of the relativistic charged particles becomes nearly equal to those obtained for the real data sets.

\section{Formalism}

For entropy of the charged particle multiplicity distribution, Shannon's information entropy is calculated using [10, 23]

$$
S=-\sum_{n} P_{n} \ln P_{n}
$$

and its generalization, Rényi's order $q$ information entropy, is estimated as $[14,26,70]$

$$
I_{q}=\frac{1}{q-1} \ln \sum_{n} P_{n}^{q} \text { for } q \neq 1,
$$

where for $q=1 \lim _{q \rightarrow 1} I_{q}=I_{1}=S$, and $P_{n}$ is the probability of production of $n$ charged particles. The generalized dimensions of order $q$ may be estimated as [14, $69,70]$

$$
\begin{aligned}
& D_{q}=\frac{I_{q}}{Y_{m}}, \\
& \quad \text { where } Y_{m}=\ln \left[\frac{\left(\sqrt{s}-2 m_{n}\left\langle n_{p}\right\rangle\right)}{m_{\pi}}\right]=\ln n_{\max } .
\end{aligned}
$$

$Y_{m}$ denotes the maximum rapidity in the centre-of-mass frame, $\sqrt{s}$ represents the center-of-mass energy, $m_{\pi}$ is the pion rest mass, $\left\langle n_{p}\right\rangle$ denotes the average number of participating nucleons, and $n_{\max }$ is maximum multiplicity of relativistic charged particles produced for a given pair of colliding nuclei at a given energy.

For the particle production process to follow self-similar behaviour, the $G$ moments of order $q$, defined as [81]

$$
G_{q}=\sum P_{n}^{q} ; \quad q \text { is any real number, }
$$

should follow the power law of the form

$$
G_{q} \propto(\partial \eta)^{\tau(q)}
$$

where $\partial \eta$ is the pseudorapidity bin-width. The parameter $\tau(q)$ is related to the dimension $D_{q}$, for all $q$, by

$$
\tau(q)=(q-1) D_{q}
$$

where $D_{0}, D_{1}$, and $D_{2}$ are usually referred to as the fractal dimensions, information dimension, and correlation dimension, respectively [41]. It has been pointed out by
Hwa [41] that values of $G_{q}$ moments obtained from various experiments can not be compared as they depend on the number of events in the data samples and on $\partial \eta$, that is, on detector resolution. It is the $\partial \eta$ dependence of $G_{q}$ that is the aim of studying $G_{q}$; in particular data analysis should aim to extract the generalized dimensions $D_{q}$, where

$$
D_{q}=(q-1)^{-1} \lim _{\partial \eta \rightarrow 0}\left(\frac{\ln G_{q}}{\ln \partial \eta}\right) .
$$

The meaning of function $\tau(q)$ becomes rather more obvious after performing the Legendre transformation from independent variables $\tau$ and $q$ to the variables $\alpha$ and $f$ :

$$
\begin{gathered}
\alpha_{q}=\frac{d \tau(q)}{d q}, \\
f\left(\alpha_{q}\right)=q \alpha_{q}-\tau(q) .
\end{gathered}
$$

$f(\alpha)$ is fractal dimension of a subset composed from bins whose occupancy probability lies in the interval $(P-d P$ to $P+d P)$. Thus by estimating the $G_{q}$ moments, the continuous scaling function $f\left(\alpha_{q}\right)$ can be constructed [44].

The thermodynamical interpretation of these relationships means that $q$ can be interpreted with an inverse temperature $q=T^{-1}$ whereas the spectrum $f(\alpha)$ and $\alpha$ play the role of entropy and energy (per unit volume), respectively [81-84].

\section{Results and Discussion}

Probability $P_{n}(\Delta \eta)$ of production of $n$ charged particles in a pseudorapidity window of fixed width is calculated by selecting a window of width $\Delta \eta=0.5$. This window is so chosen that its midposition coincides with the center of symmetry of $\eta$ distribution, $\eta_{c}$. Thus, all the relativistic charged particles having their $\eta$ values lying in the range $\left(\eta_{c}-\Delta \eta / 2\right) \leq \eta \leq\left(\eta_{c}+\Delta \eta / 2\right)$ are counted to evaluate $P_{n}$. The window size is then increased in steps of 0.5 until the region, $\eta_{c} \pm 3.0$, is covered. Values of entropy, $S$, for different $\eta$-windows are calculated by using (2), while the value of maximum rapidity is estimated from (4). Variations of entropy normalized to maximum rapidity, $S / Y_{m}$, with $\eta$-window width, also normalized to maximum rapidity, $\Delta \eta / Y_{m}$, for the experimental and HIJING data sets are plotted in Figure 1. It is observed in the figure that $S / Y_{m}$ first increases up to $\Delta \eta / Y_{m} \sim 0.5$ and thereafter acquires nearly a constant value. It is interesting to note that the data points for various samples of events overlap to form a single curve. This indicates the presence of entropy scaling in AA collisions at AGS and SPS energies. Results from HIJING simulated events also support the presence of entropy scaling.

Similar entropy scaling in AA collisions has been reported by us $[10,15]$ and also by the other workers $[29,70]$ for central and minimum bias events. In our earlier work, attempt was made to look for whether the observed entropy scaling is of dynamical nature. For this purpose the correlation free Monte Carlo events samples (Mixed events) corresponding to each of the real data samples were generated and analyzed. These 


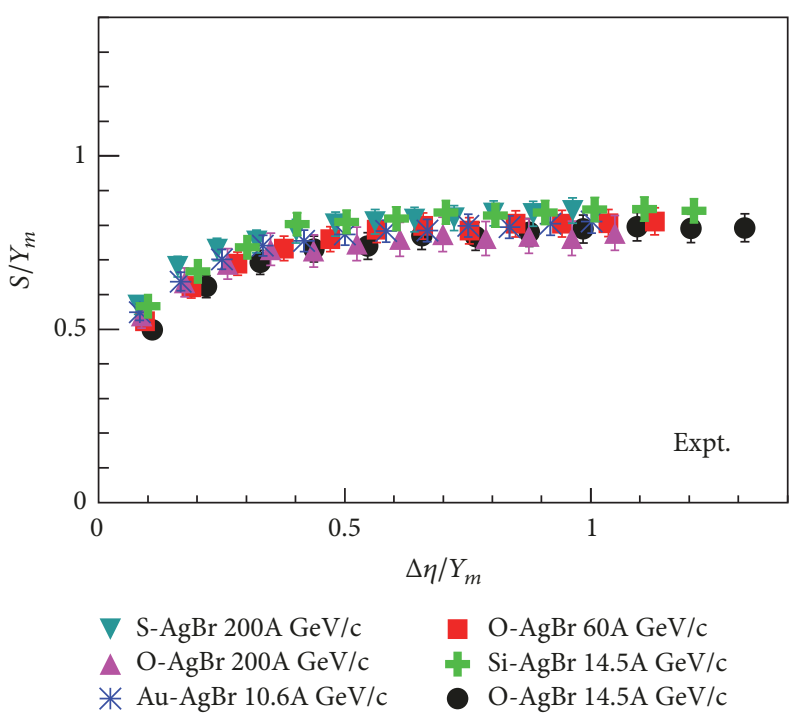

(a)

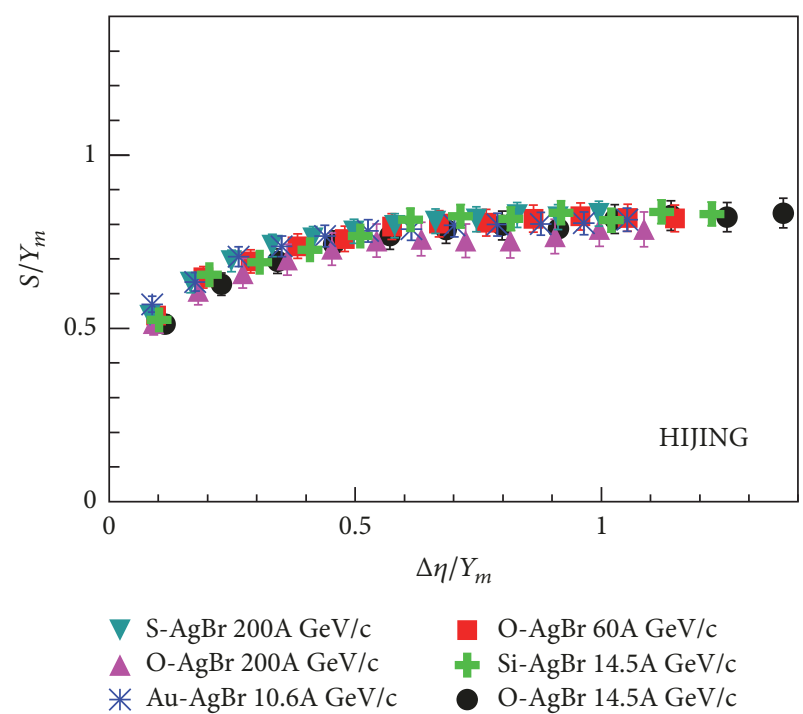

(b)

FIGURE 1: Variations of entropy normalized to maximum rapidity, $S / Y_{m}$, with $\eta$-window width normalized to maximum rapidity, $\Delta \eta / Y_{m}$, for the Experimental (a) and HIJING events (b).

findings revealed that the entropy scaling observed was the distinct feature of the data and was of dynamical origin [10, 15].

According to (4) the quantity, $\left(\sqrt{s}-2 m_{n}\left\langle n_{p}\right\rangle\right) / m_{\pi}$, is equal to the maximum charged particle multiplicity at a given center of mass energy. It would, therefore, be convenient to examine the mean multiplicity $\left\langle n_{s}\right\rangle$ versus entropy in limited $\eta$ windows as well as in full $\eta$ range; the entropy in the entire $\eta$ range, $S_{\max }$, is calculated using (2). Variations of $S_{\max }$ with $\ln E_{\text {total }}$ for the experimental and HIJING events are exhibited in Figure 2: $E_{\text {total }}$ denotes the total energy of the beam nucleus. It may be noticed in the figure that $S_{\max }$ increases linearly with $\ln E_{\text {total }}$ for both real and simulated event samples. HIJING, however, predicts somewhat smaller values of $S_{\max }$ as compared to the corresponding $S_{\max }$ values estimated from the real data. These findings are, thus, in agreement with those reported for ${ }^{16} \mathrm{O}$-nucleus collision at $60 \mathrm{~A}$ and $200 \mathrm{~A} \mathrm{GeV} / \mathrm{c}$ and ${ }^{32} \mathrm{~S}$-nucleus interaction at $200 \mathrm{GeV} / \mathrm{c}[70]$. Shown in Figure 3 are the variations of $S / S_{\max }$ with $\langle n\rangle /\langle n\rangle_{\max }$ for the six data sets considered. It is evident from the figure that the data points corresponding to various types of collisions almost overlap to form a single curve. It may also be noted that $S / S_{\max } \rightarrow 1.0$ as $\langle n\rangle /\langle n\rangle_{\max } \rightarrow$ 1.0. These observations too support the presence of entropy scaling in AA collisions at AGS and SPS energies.

It has been reported $[14,81,85,86]$ that the constant specific heat $(\mathrm{CSH})$ approximation widely used in standard thermodynamics is applicable to the multifractal data too. Nearly constant value of multifractal specific heat has been observed [87-90] from the analysis of the experimental data on hadronic and heavy-ion collisions at different energies. These investigations have been carried out by following the methods proposed by Hwa [41] and Takagi [88], where the multiplicities of particles in narrow $\eta$-bins, $\delta \eta$, are involved. This introduces a limit to analysis because $\delta \eta$ would depend

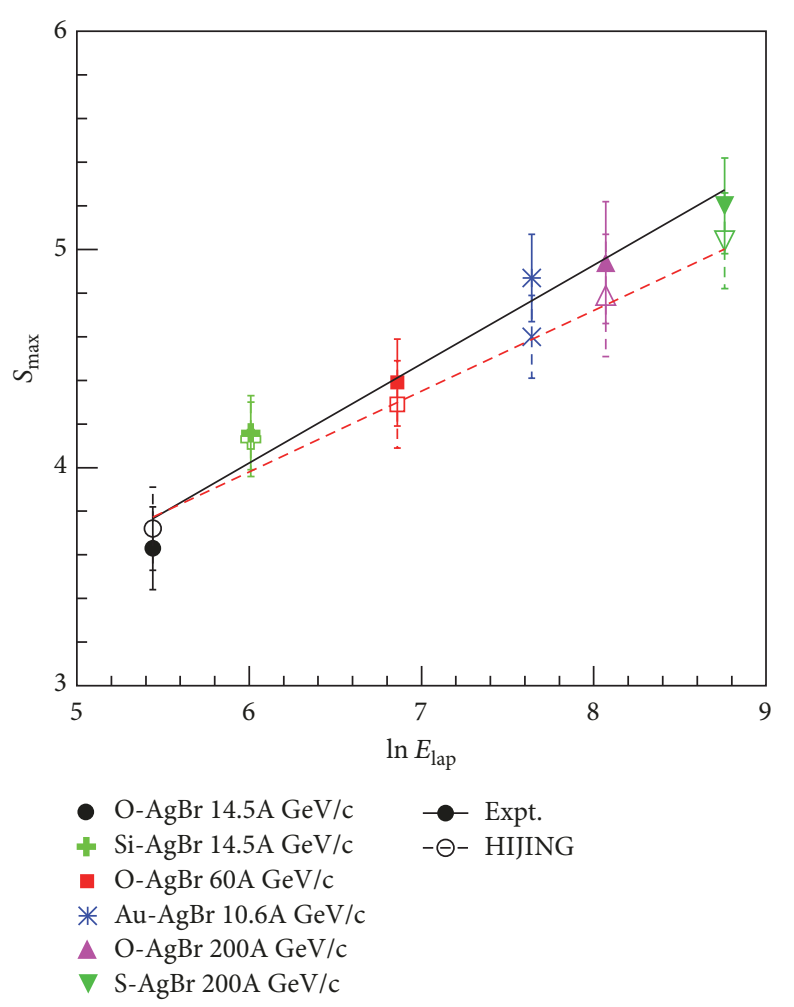

FIGURE 2: Variations of $S_{\max }$ with $\ln E_{\text {total }}$ for the experimental and HIJING events.

on the detector resolution (see (6)). However, since the quantity $\sum\left(P_{n}\right)^{q}$ scales with improving relative resolution $\delta \eta$ [70] like $G_{q} \approx(\delta \eta)^{\tau(q)}=\sum\left(P_{n}\right)^{q}$, the Rényi's order$q$ information entropy may also be used to understand the multifractal nature of particle production and estimation 

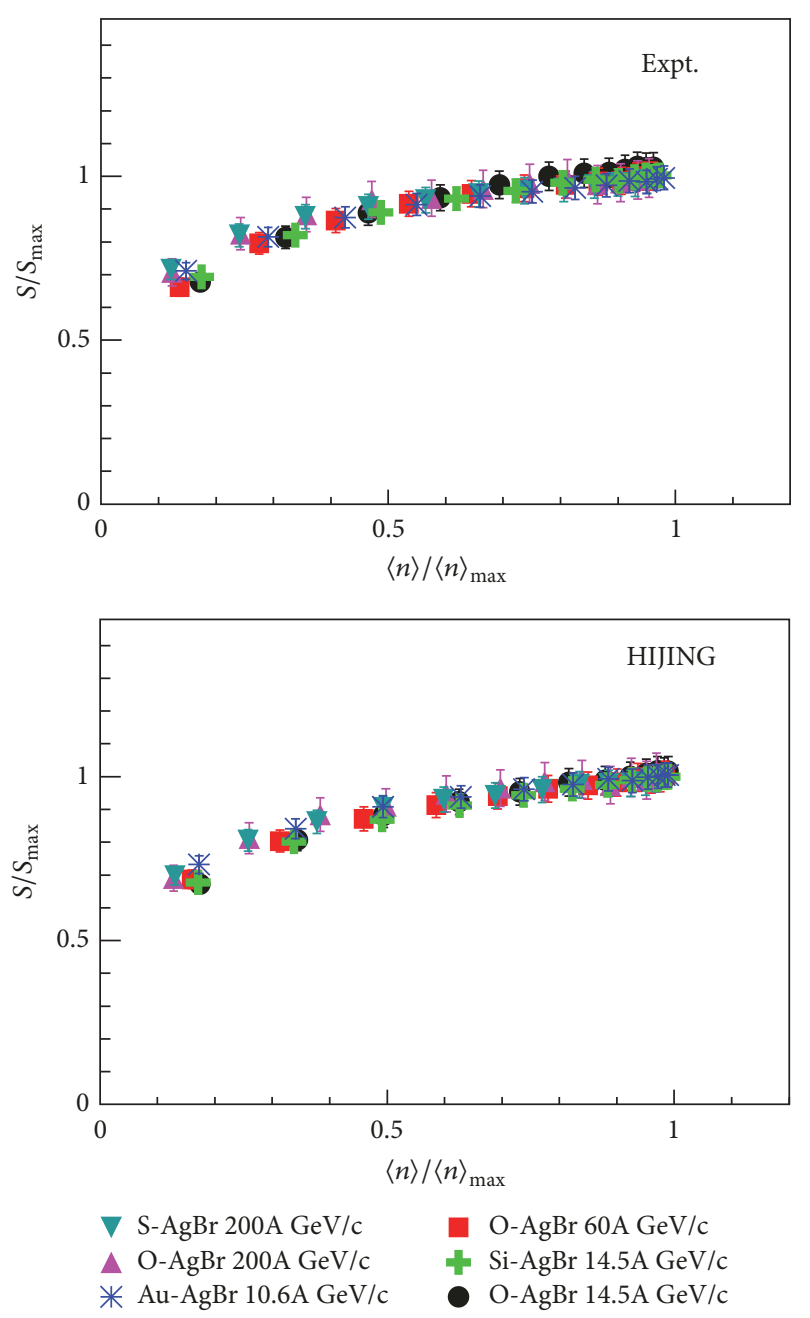

Figure 3: Variations of $S / S_{\max }$ with $\langle n\rangle /\langle n\rangle_{\max }$ for the experimental and HIJING event samples.

of multifractal specific heat. The advantage of this method is that it does not depend on the phase space bin width and hence on detector resolution; rather it accounts for the fractal resolution, which is related to the collision energy [14]. From the definitions of Rényi's information entropy, $I$, and generalized dimensions, $D_{q}$ (see (3) and (4)), it is evident that, for a given $q,\left(I_{q}\right)_{\max }=\ln n_{\max }$. The highest entropy is achieved for the greatest "chaos" of a uniformly distributed probability function $P_{n}=1 / n_{\max }$ [71] and (4) gives $D_{q}=I_{q} /\left(I_{q}\right)_{\max }$.

Variations of $D_{q}$ with $q$ for various event samples are shown in Figure 4 . The values of $D_{1}$ have been obtained from the $f\left(\alpha_{q}\right)$ spectrum (Figure 6) and discussed in the coming part of the text. It is observed that $D_{q}$ monotonously decreases with increasing order $q$ and the trend of decrease for the real and HIJING events is nearly the same except that HIJING predicts somewhat smaller values of $D_{q}$ as compared to that for experimental data. The $D_{q}$ spectrum for order $q \geq 2$, which for multifractals is decreasing function of $q$, can be related to the scaling behaviour of $q$ point correlation integrals [14, 47]. Thus, the trend of variations of $D_{q}$ against $q$ observed in the present study indicates the
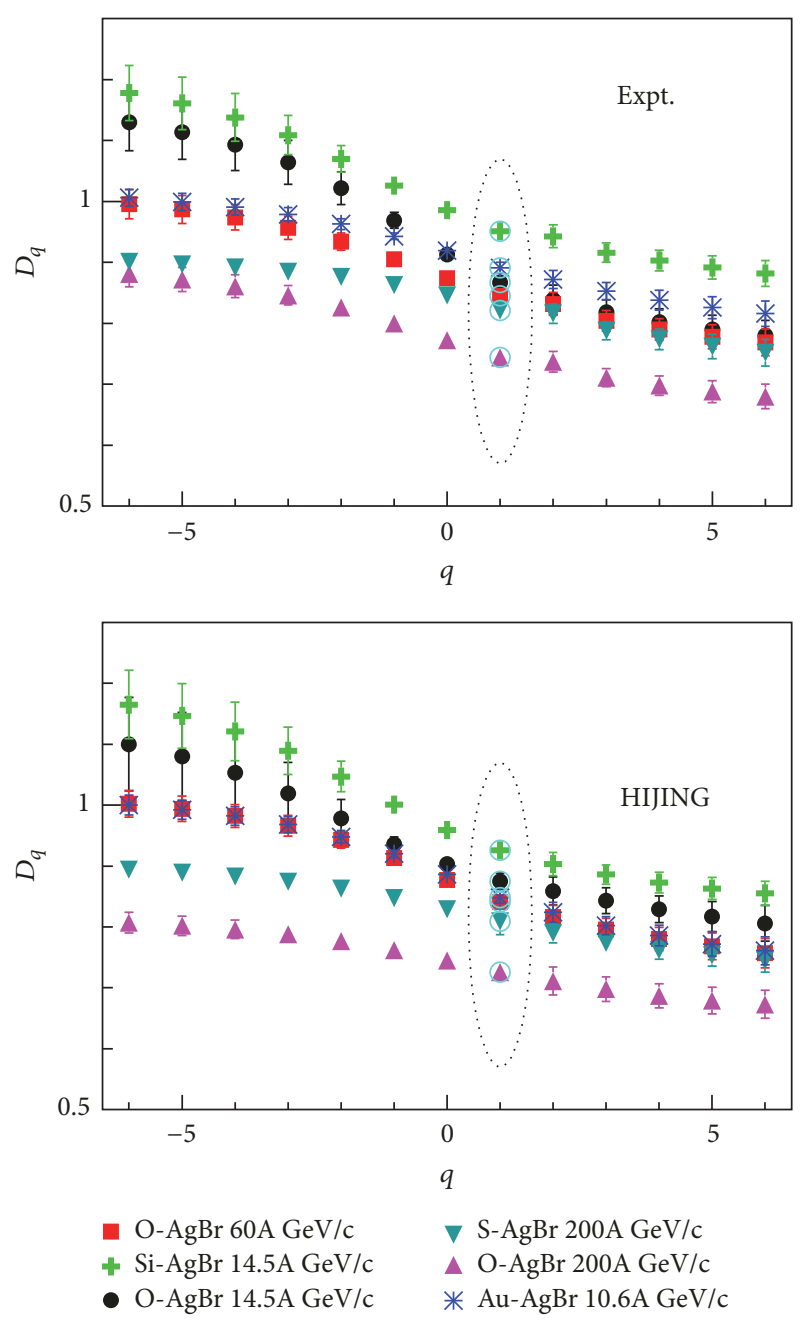

FIgURE 4: Dependence of $D_{q}$ on $q$ for experimental and HIJING events. The values of $D_{1}$ (encircled) are obtained from $f(\alpha(q))$ spectrum.

multifractal nature of multiplicity distributions in full phase space in ion-ion collisions at the energies considered. Similar variations of $D_{q}$ with $q$ have also been reported earlier [70] in collisions of protons $(800 \mathrm{GeV})$ and heavy ions $\left({ }^{4} \mathrm{He}\right.$ at $11 \mathrm{~A}$, ${ }^{28} \mathrm{Si}$ at $14.5 \mathrm{~A},{ }^{16} \mathrm{O}$ at $60 \mathrm{~A}$ and $200 \mathrm{~A}$, and ${ }^{32} \mathrm{~S}$ at $200 \mathrm{~A} \mathrm{GeV} / \mathrm{c}$ ) collisions. Although the presence of multifractality predicts the decrease of $D_{q}$ with $q$, yet no further useful information about the $q$-dependence of $D_{q}$ spectrum can be extracted which may lead to making remarks on the scaling properties of $q$-correlation integrals [14]. It has been suggested [81, 85, 90] that in constant heat approximation, $D_{q}$, dependence on $q$ acquires the following simple form:

$$
D_{q} \simeq(a-c)+c \frac{\ln q}{q-1},
$$

where $a$ is the information dimension, $D_{1}$, while $c$ denotes the multifractal specific heat. The linear trend of variation $D_{q}$ with $\ln q / q-1$, given by (10), is expected to be observed for multifractals. On the basis of classical analogy with specific 
TABLE 2: Values of parameters $a$ and $c$, occurring in (4) for the various event samples.

\begin{tabular}{|c|c|c|c|c|c|}
\hline \multirow{2}{*}{ Type of interactions } & \multirow{2}{*}{ Energy $(\mathrm{GeV} / \mathrm{c})$} & \multicolumn{2}{|c|}{ Expt. } & \multicolumn{2}{|c|}{ HIJING } \\
\hline & & $a$ & $c$ & $a$ & $c$ \\
\hline${ }^{197} \mathrm{Au}-\mathrm{AgBr}$ & $10.6 \mathrm{~A}$ & $0.746 \pm 0.003$ & $0.195 \pm 0.006$ & $0.684 \pm 0.002$ & $0.213 \pm 0.004$ \\
\hline${ }^{16} \mathrm{O}-\mathrm{AgBr}$ & $14.5 \mathrm{~A}$ & $0.708 \pm 0.001$ & $0.200 \pm 0.003$ & $0.738 \pm 0.003$ & $0.189 \pm 0.006$ \\
\hline${ }^{28} \mathrm{Si}-\mathrm{AgBr}$ & $14.5 \mathrm{~A}$ & $0.815 \pm 0.002$ & $0.185 \pm 0.005$ & $0.795 \pm 0.002$ & $0.167 \pm 0.004$ \\
\hline${ }^{16} \mathrm{O}-\mathrm{AgBr}$ & $60 \mathrm{~A}$ & $0.700 \pm 0.002$ & $0.191 \pm 0.004$ & $0.682 \pm 0.002$ & $0.209 \pm 0.005$ \\
\hline${ }^{16} \mathrm{O}-\mathrm{AgBr}$ & $200 \mathrm{~A}$ & $0.613 \pm 0.001$ & $0.190 \pm 0.003$ & $0.614 \pm 0.001$ & $0.158 \pm 0.004$ \\
\hline${ }^{32} \mathrm{~S}-\mathrm{AgBr}$ & $200 \mathrm{~A}$ & $0.681 \pm 0.002$ & $0.196 \pm 0.005$ & $0.685 \pm 0.001$ & $0.165 \pm 0.003$ \\
\hline
\end{tabular}
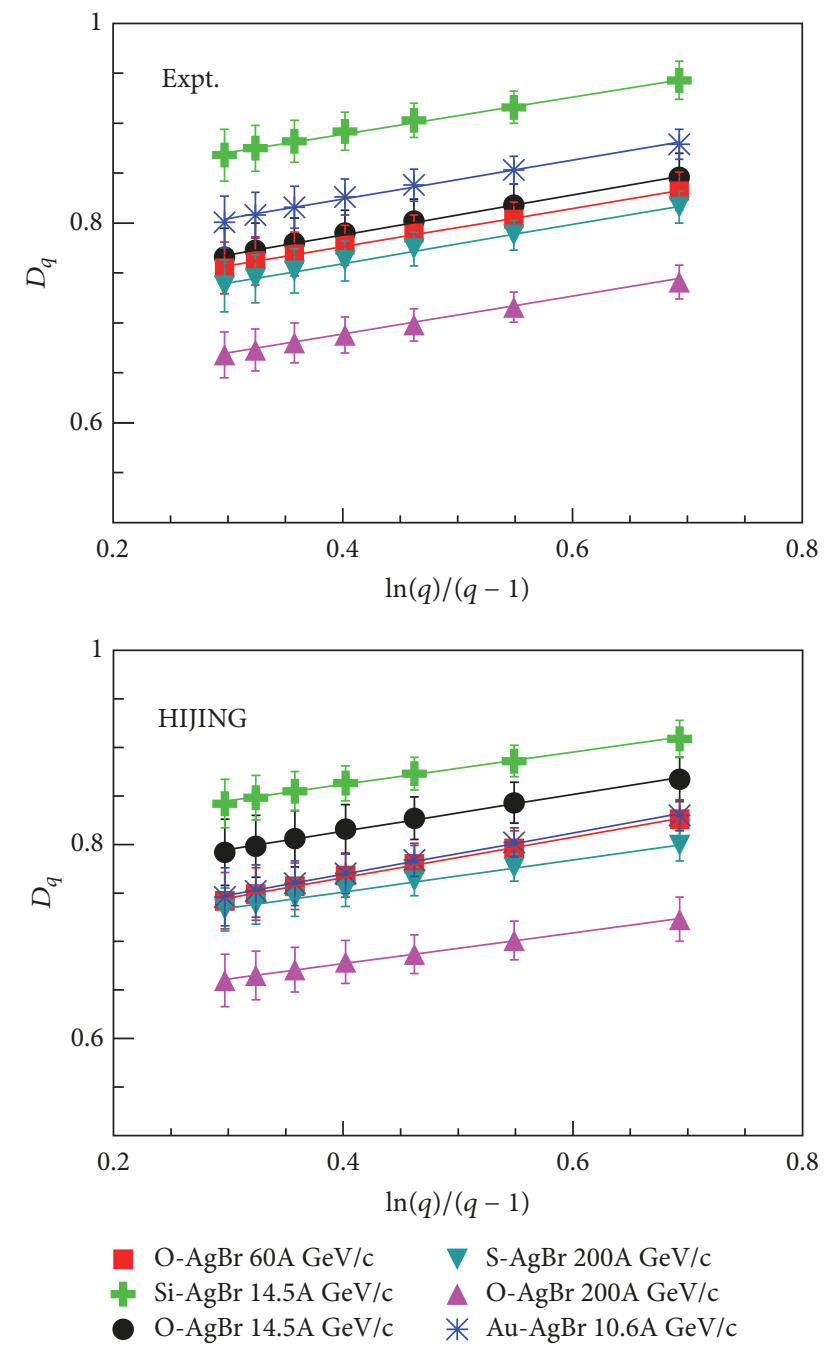

Figure 5: $D_{q}$ versus $\ln (q) /(q-1)$ plots for the experimental and HIJING data at various energies.

heat of gases and solids, the value of $c$ is predicted [89] to remain independent of temperature in a wide range of $q$. In order to test the validity of (10), $D_{q}$ values are plotted against $\ln q / q-1$ in Figure 5. It is evident from the figure that $D_{q}$ increases linearly with $\ln q / q-1$. The lines in the figure are due to the best fits to the data obtained using (10). The values of the parameters " $a$ " and " $c$," thus, obtained are listed in Table 2. It is interesting to note from the table that the values of multifractal specific heat, $c$, for all the data sets, are nearly the same, $\sim 0.2$ there by indicating its independence of the beam energy and projectile mass. It may also be noticed from the table that the experimental values of $c$ are quite close to those predicted by HIJING. It is worthwhile to mention that the values of multifractal specific heat obtained in the present study are close to those obtained by us [87] by analysing some of these data sets using Takagi's approach [88]. Incidentally similar values of $c$ have been reported by $\mathrm{Du}$ et al. [90] for $10.6 \mathrm{~A} \mathrm{GeV} / \mathrm{c}{ }^{197} \mathrm{Au}$ nucleus collisions. In $p$-nucleus interactions too, the value of multifractal specific heat has been observed to be $\sim 0.25$ in the energy range, $200-800 \mathrm{GeV}[70,85,90]$. However, in the case of $p p / \bar{p} p$ collisions the values of this parameter have been found to be $\sim 0.08$ in the wide energy range $25-1800 \mathrm{GeV}$ [14].

These findings, thus, indicate that the constant-specific heat approximation is applicable to the multiparticle production in relativistic hadronic and ion-ion collisions. Moreover, nearly the same values of multifractal specific heat, $c$, observed in the present study as well as other workers using the data on hh, hA, and AA collisions involving various projectiles/targets at widely different energies do indicate that the parameter $c$ may be regarded as a universal characteristics of hadronic and heavy-ion collisions.

In order to construct the multifractal spectrum, values of $\tau_{q}, \alpha_{q}$, and $f\left(\alpha_{q}\right)$ are evaluated using (7) and (9) for $q=$ -6 to 6. $f\left(\alpha_{q}\right)$ spectra for various data sets are displayed in Figure 6. It is evidently clear from the figure that these spectra are represented by continuous curves, thus, characterising a qualitative manifestation of the multiplicity fluctuations. Spectra for all the data sets (real and HIJING ) are noticed to follow the general characteristics of occurrence of peaks at $\alpha_{0}$ and a common target at an angle of $45^{\circ}$ at $f\left(\alpha_{1}\right)=$ $\alpha_{1}$. The spectrum is noticed to have a peak at $\alpha_{0}$ and is concave downwards everywhere. As expected the values of $\alpha_{q}$ decrease with increasing $q$ as $D_{q}$ decreases with increasing $q$. The region $\alpha_{q}<\alpha_{0}$ corresponds to positive $q$ and the curves in this region have positive slope, whereas the region $\alpha_{q}>\alpha_{0}$ would correspond to negative $q$ and the slope of the curves in this region is negative.

The values of $f\left(\alpha_{q}\right)$ for $q=0,1,2$ would give the fractal dimensions $\left(D_{0}=f\left(\alpha_{0}\right)\right)$, the information dimension $\left(D_{1}=f\left(\alpha_{1}\right)=\alpha_{1}\right)$, and the correlation dimension $\left(D_{2}=\right.$ $\left.2 \alpha_{2}-f\left(\alpha_{2}\right)\right)$. The values of information dimension are shown in Figure 4 to distinguish these points in the figure; the 
TABLE 3: Values of width $\left(\alpha_{\max }-\alpha_{\min }\right)$ of $f(\alpha(q))$ spectrum for the various event samples.

\begin{tabular}{lccccccc}
\hline Type of interactions & Energy $(\mathrm{GeV} / \mathrm{c})$ & $\alpha_{\max }$ & $\alpha_{\min }$ & $\left(\alpha_{\max }-\alpha_{\min }\right)$ & $\alpha_{\max }$ & \multicolumn{2}{c}{ HIJING } \\
& & 1.045 & 0.776 & 0.269 & 1.045 & 0.716 \\
${ }^{197} \mathrm{Au}-\mathrm{AgBr}$ & $10.6 \mathrm{~A}$ & 1.224 & 0.742 & 0.482 & 1.220 & 0.764 & 0.329 \\
${ }^{16} \mathrm{O}-\mathrm{AgBr}$ & $14.5 \mathrm{~A}$ & 1.279 & 0.845 & 0.434 & 1.278 & 0.821 & 0.456 \\
${ }^{28} \mathrm{Si}-\mathrm{AgBr}$ & $14.5 \mathrm{~A}$ & 1.054 & 0.732 & 0.322 & 1.055 & 0.717 & 0.457 \\
${ }^{16} \mathrm{O}-\mathrm{AgBr}$ & $60 \mathrm{~A}$ & 0.932 & 0.647 & 0.285 & 0.834 & 0.647 & 0.187 \\
${ }^{16} \mathrm{O}-\mathrm{AgBr}$ & $200 \mathrm{~A}$ & 0.924 & 0.712 & 0.212 & 0.923 & 0.713 \\
${ }^{32} \mathrm{~S}-\mathrm{AgBr}$ & $200 \mathrm{~A}$ & & & & & 0.210 \\
\hline
\end{tabular}
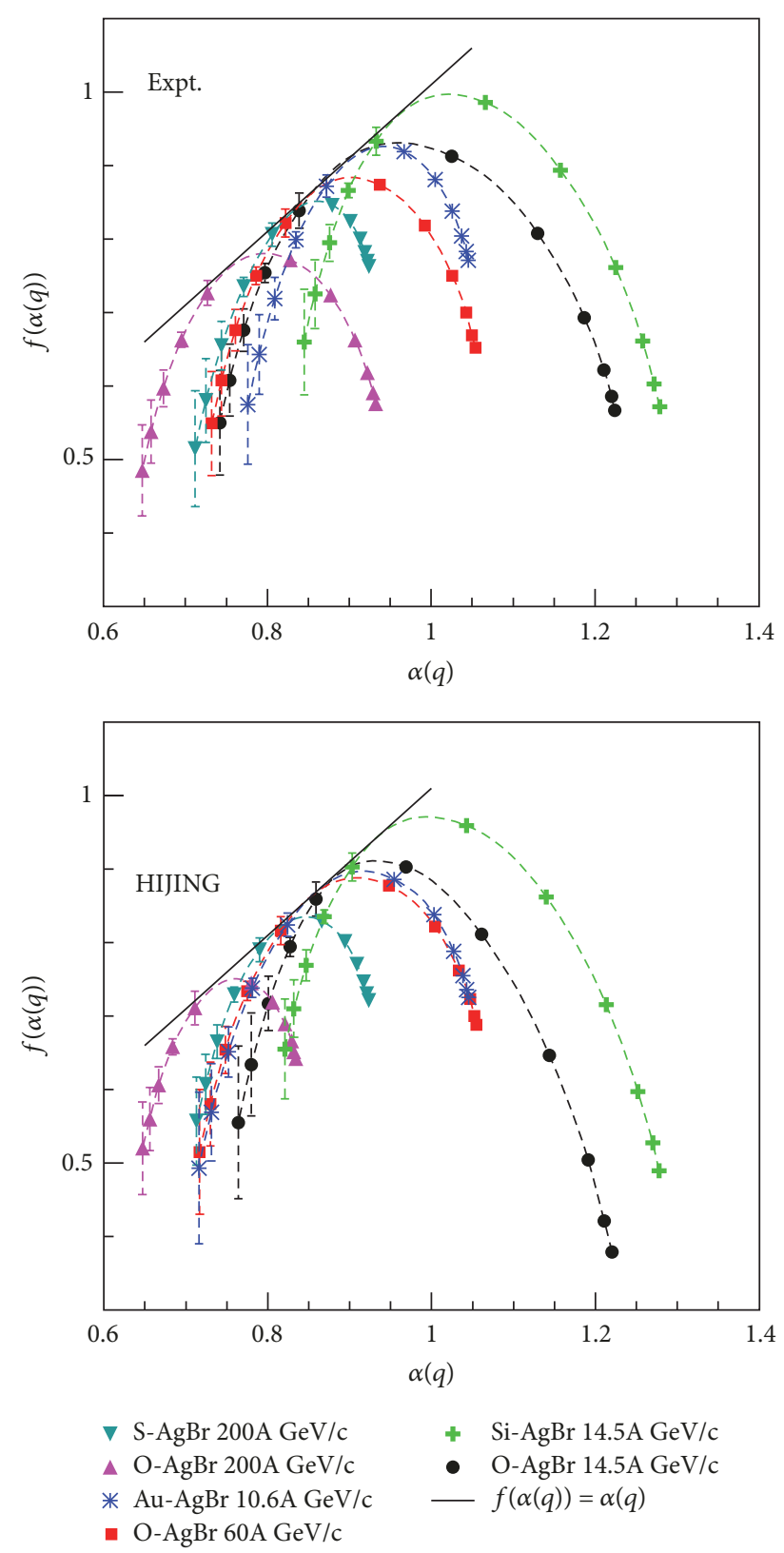

FIGURE 6: $f(\alpha(q))$ spectra for the experimental and HIJING event samples. data points are encircled. The widths of the $f\left(\alpha_{q}\right)$ spectrum $\left(\alpha_{\max }-\alpha_{\min }\right)$ for various data sets (real and HIJING ) are also calculated and presented in Table 3. The width of the spectrum has been suggested as a measure of the degree of multifractality [91-93]. The broader the spectrum, the higher the multifractality $[93,94]$.

It may be observed from Figure 6 that the spectrum becomes wider with increasing beam energy $\left({ }^{16} \mathrm{O}-\mathrm{AgBr}\right.$ data at $14.5 \mathrm{~A}, 60 \mathrm{~A}$, and $200 \mathrm{~A} \mathrm{GeV} / \mathrm{c}$ ) as well as with increasing projectile mass $\left({ }^{16} \mathrm{O}-\mathrm{AgBr}\right.$ and ${ }^{28} \mathrm{Si}-\mathrm{AgBr}$ data at $14.5 \mathrm{~A} \mathrm{GeV} / \mathrm{c}$ and ${ }^{16} \mathrm{O}-\mathrm{AgBr}$ and ${ }^{32} \mathrm{~S}-\mathrm{AgBr}$ data at $\left.200 \mathrm{~A} \mathrm{GeV} / \mathrm{c}\right)$. Similar dependence of $f\left(\alpha_{q}\right)$ spectrum has also been observed in ${ }^{12} \mathrm{C}$-, ${ }^{24} \mathrm{Mg}-,{ }^{16} \mathrm{O}-$, and ${ }^{32} \mathrm{~S}-\mathrm{AgBr}$ collisions at $2.1 \mathrm{~A}, 4.5 \mathrm{~A}, 60 \mathrm{~A}$, and $200 \mathrm{~A} \mathrm{GeV/c} \mathrm{[93].}$

These observations, thus, tend to suggest that multifractality is more pronounced if the beam energy or mass of the colliding nuclei increases. However, it has been pointed out that at higher energies influence of energy is more prominent as compared to the mass [93]. Hwa [41] has also pointed out that since $D_{q}$ increases with energy for all $q$ values, $\alpha_{0}$ and $f\left(\alpha_{0}\right)$ will increase with energy too and hence the entire spectrum $\left(f\left(\alpha_{q}\right)\right)$ would be a broader once. This, in turn, implies that the rapidity distribution will become more jagged and irregular with prominent sharp peaks and deep valleys. As the analysis method suggested by Hwa [41] is based on the multiplicity fluctuations in limited rapidity bins, the remark made in [41] that the highly chaotic behaviour of MD in narrow rapidity bins, $n(y)$, can be observed by a smooth function $f(\alpha)$ is quite interesting. It has also been remarked that averaging $n(y)$ over all events would be devoid of any information about fluctuation and intermittency and also that if the experimental/detector resolution is not good enough to capture the sharp peaks and deep valleys, the $f\left(\alpha_{q}\right)$ spectrum would appear narrower than it should otherwise be [41]. In contrast to the standard intermittency and multifractal analysis which are based on the multiplicities in phase space bins, the present analysis, as mentioned earlier, does not depend on the experimental resolution. It rather considers fractal resolution related to the total energy available [14]. Assuming that, during collisions with many particles produced, energy dissipates into $N$ discrete sites. The site labeled by $n$ is occupied by the probability $P_{n}$. Since most of the sites are unoccupied, the overlay of many inelastic 
events can be visualized as a fractal with overall extent $\sqrt{s}$. The sufficient condition to produce self-similar structure is that $P_{n}$ would exhibit some type of scaled invariant behaviour. At sufficiently high energies, which correspond to high enough resolution $\delta=1 / N, P_{n}$ acquires a power law dependence on the resolution $\delta$ and hence the quantity $\sum\left(P_{n}\right)^{q}$ will scale with $\delta$ as $[14]$

$$
\sum\left(P_{n}\right)^{q} \sim(\delta)^{-(1-q) D}
$$

The independence of generalized dimension

$$
D_{q}=-\frac{I_{q}}{n \delta}
$$

on energy (resolution $\delta$ ) would refer the presence of multifractality in $\mathrm{MD}$ of relativistic charged particles produced in high energy collisions [14, 95].

\section{Conclusions}

On the basis of the findings of the present work the following conclusions may be reached:

(1) The entropy normalized to maximum rapidity exhibits a saturation beyond $\Delta \eta / Y_{m} \sim 0.5$ indicating thereby the presence of large amount of entropy around midrapidity region.

(2) Overlapping data points for various sets of events in $S / Y_{m}$ vs $\Delta \eta / Y_{m}$ plots exhibit entropy scaling in AA collisions at AGS and SPS energies.

(3) The scaling observed with the experimental data is nicely supported by HIJING model.

(4) The decreasing values of $D_{q}$ with increasing order number $q$ may be taken as a signal of multifractal nature of multiplicity distributions of relativistic charged particle produced in hadronic and heavy-ion collisions.

(5) Besides the information dimension, $D_{1}$, there is yet another parameter, $c$, which may be used as a universal parameter for multiparticle production in high energy hadronic and heavy-ion collisions.

(6) Rényi's order $q$-information entropy may also be used to construct the multifractal spectrum, which in turn would help study the multiplicity fluctuations and scaling in high energy hadronic and heavy-ion collisions.

\section{Conflicts of Interest}

The authors declare that there are no conflicts of interest.

\section{References}

[1] M. Mukherjee, S. Basu, S. Choudhury, and T. K. Nayak, "Fluctuations in charged particle multiplicities in relativistic heavy-ion collisions," Journal of Physics G, vol. 43, no. 8, Article ID 085102, 2016.
[2] A. Kumar, P. K. Srivastava, B. K. Singh, and C. P. Singh, "Charged hadron multiplicity distribution at relativistic heavyion colliders," Advances in High Energy Physics, vol. 2013, pp. $1-27,2013$.

[3] A. Alkin, "Phenomenology of charged-particle multiplicity distributions," Ukrainian Journal of Physics, vol. 62, pp. 743-756, 2017.

[4] I. M. Dremin and J. W. Gary, "Hadron multiplicities," Physics Reports, vol. 349, p. 301, 2001.

[5] P. Castorina, G. Nardulli, and G. Preparata, "Are quantumchromodynamic scaling violations in deep-inelastic leptonhadron scattering really observed?" Physical Review Letters, vol. 47, no. 7, pp. 468-471, 1981.

[6] G. Hanson, "Evidence for jet structure in hadron production by $e^{+} e^{-}$annihilation," Physical Review Letters, vol. 35, no. 24, pp. $1607-1609,1975$.

[7] C. Berger, "A study of jets in electron positron annihilation into hadrons in the energy range 3.1 to $9.5 \mathrm{GeV}$," Physics Letters $B$, vol. 78B, no. 1, pp. 176-182, 1978.

[8] G. Aad et al., "Measurement of multi-jet cross sections in proton-proton collisions at a $7 \mathrm{TeV}$ center-of-mass energy," The European Physical Journal C, vol. 71, p. 1763, 2011.

[9] Z. Bern, "Two "New" categories of papers welcomed in corrosion," Corrosion, vol. 68, no. 4, 2012.

[10] S. Ahmad, A. Chandra, M. Zafar, M. Irfan, and A. Ahmad, "Entropy scaling in ion-ion collisions at ags and sps energies," International Journal of Modern Physics E, vol. 22, no. 8, Article ID 1330019, 2013.

[11] S. Das, S. K. Ghosh, S. Raha, and R. Ray, "Entropy scaling from chaotically produced particles in p-p collisions at LHC energies," Nuclear Physical A, vol. 438, pp. 862-863, 2011.

[12] R. M. Weiner, "Primordial nucleosynthesis: successes and challenges," International Journal of Modern Physics E, vol. 15, no. 1, p. 37, 2006.

[13] S. Das, S. K. Ghosh, S. Raha, and R. Ray, "Entropy scaling and thermalization in hadron-hadron collisions at LHC," High Energy Physics - Phenomenology, p. 4, 2011.

[14] M. K. Suleymanov, M. Sumbera, and I. Zborovsky, "hep$\mathrm{ph} / 0304206$ ".

[15] S. Ahmad, A. Ahmad, A. Chandra, M. Zafar, and M. Irfan, "Entropy analysis in relativistic heavy-ion collisions," Advances in High Energy Physics, vol. 2013, Article ID 836071, 10 pages, 2013.

[16] G. J. Alner, "Scaling violation favouring high multiplicity events at 540 GeV CMS energy," Physics Letters B, vol. 138B, pp. 304310,1984

[17] Z. Koba, H. B. Nielsen, and P. Olesen, "Scaling of multiplicity distributions in high energy hadron collisions," Nuclear Physics $B$, vol. 40, pp. 317-334, 1972.

[18] G. J. Alner, "Scaling violations in multiplicity distributions at 200 and 900 GeV," Physics Letters B, vol. 167B, pp. 476-480, 1986.

[19] A. Wroblewski, "Multiplicity distributions in proton proton collisions," Acta Physica Polonica B, vol. 4, p. 857, 1973.

[20] W. Thome, K. Eggert, and K. Giboni, "Charged particle multiplicity distributions in pp collisions at ISR energies," Nuclear Physics B, vol. 129, no. 3, pp. 365-389, 1977.

[21] G. J. Alner, "A new empirical regularity for multiplicity distributions in place of KNO scaling," Physics Letters B, pp. 199-206, 1985. 
[22] G. J. Alner, "Multiplicity distributions in different pseudorapidity intervals at a CMS energy of $540 \mathrm{GeV}$," Physics Letters B, vol. 160B, pp. 193-198, 1985.

[23] V. Simak, M. Sumbera, and I. Zborovsky, "Entropy in multiparticle production and ultimate multiplicity scaling," Physics Letters B, vol. 206, no. 1, pp. 159-162, 1988.

[24] Y. M. Sinyukov and S. V. Akkelin, "Pion entropy and phasespace densities in $A+A$ collisions," Acta Physica Hungarica Series A, vol. 22, no. 1-2, pp. 171-178, 2005.

[25] S. Pal and S. Pratt, "Entropy production at RHIC," Physics Letters $B$, vol. 578, no. 3-4, pp. 310-317, 2004.

[26] P. Carruthers, M. Plümer, S. Raha, and R. Weiner, "Entropy scaling in high energy collisions. Chaos and coherence," Physics Letters B, vol. 212, no. 3, pp. 369-374, 1988.

[27] M. R. Ataian, "EHS/NA22 collaboration," Acta Physica Polonica $B$, vol. 36, p. 2969, 2005.

[28] T. Mizoguchi and M. Biyajima, "Analyses of multiplicity distributions with $\eta_{c}$ and Bose-Einstein correlations at LHC by means of generalized Glauber-Lachs formula," The European Physical Journal C, vol. 70, no. 4, pp. 1061-1069, 2010.

[29] D. Ghosh et al., "Entropy scaling in high-energy nucleusnucleus interactions," Journal of Physics G, vol. 38, no. 065105, 2011.

[30] D. Ghosh, A. Deb, P. K. Haldar, S. R. Sahoo, and D. Maity, "Validity of the negative binomial multiplicity distribution in case of ultra-relativistic nucleus-nucleus interaction in different azimuthal bins," Europhysics Letters, vol. 65, p. 311, 2004.

[31] T. H. Burnett, S. Dake, M. Fuki et al., "Extremely high multiplicities in high-energy nucleus-nucleus collisions," Physical Review Letters, vol. 50, no. 26, 2062 pages, 1983.

[32] G. J. Alner, R. E. Ansorge, and B. Åsman, "Scaling of pseudorapidity distributions at c.m. energies up to $0.9 \mathrm{TeV}$," Zeitschrift für Physik C: Particles and Fields, vol. 33, no. 1, pp. 1-6, 1986.

[33] M. Adamus, "Maximum particle densities in rapidity space of $\pi$ $+p, K+p$ and $p p$ collisions at $250 \mathrm{GeV} / c$," Physics Letters B, vol. 185, no. 3-4, p. 446, 1987.

[34] R. Holynski et al., "Evidence for intermittent patterns of fluctuations in particle production in high-energy interactions in nuclear emulsion," Physical Review Letters, vol. 62, p. 733, 1989.

[35] A. Bialas and R. Peschanski, "Moments of rapidity distributions as a measure of short-range fluctuations in high-energy collisions," Nuclear Physics B, vol. 273, no. 3-4, pp. 703-718, 1986.

[36] D. Ghosh, "Evidence of non-statistical fluctuation in mediumenergy protons emitted in the fragmentation process of ultrarelativistic nuclear interaction," Europhysics Physical Letters, vol. 48, p. $508,1999$.

[37] D. Ghosh, "Multifractality and intermittency study of ultrarelativistic ${ }^{32} \mathrm{~S}-\mathrm{AgBr} \mathrm{AND}^{16} \mathrm{O}-\mathrm{AgBr}$ interactions: further evidence of a nonthermal phase transition," International Journal of Modern Physics A, vol. 14, p. 2091, 1999.

[38] M. I. Adamovich, "Scaled-factorial-moment analysis of 200AGeV sulfur+gold interactions," Physical Review Letters, vol. 65, no. 6 , p. 412, 1990.

[39] M. M. Khan, A. Shakeel, N. Ahmad et al., "Dynamical fluctuations and Levy stability in $14.5-\mathrm{A}-\mathrm{GeV} / \mathrm{c} \mathrm{Si}-28$ nucleus interactions," Acta Physica Polonica B, vol. 38, p. 2653, 2007.

[40] A. Bialas and R. B. Peschanski, "Intermittency in multiparticle production at high energy," Nuclear Physics B, vol. 308, p. 857, 1988.
[41] R. C. Hwa, "Fractal measures in multiparticle production," Physical Review D: Particles, Fields, Gravitation and Cosmology, vol. 41, no. 5, pp. 1456-1462, 1990.

[42] B. Buschbeck, P. Lipa, and R. Peschanski, "Signal for intermittency in e+e- reactions obtained from negative binomial fits," Physics Letters B, vol. 215, no. 4, pp. 788-791, 1988.

[43] I. V. Ajinenko, "Results and future prospects for muon $(g-2)$," Physics Letters B, vol. 222, no. 306, 1989.

[44] P. L. Jain, G. Singh, and A. Mukhopadhyay, "Multifractals at relativistic energies," Physical Review C: Nuclear Physics, vol. 46, no. 2, pp. 721-726, 1992.

[45] B. B. Mandelbrot, The Fractal Geometry of Nature, Freeman, New York, NY, USA, 1982.

[46] R. Saha, A. Deb, D. Ghosh, and J. Phys, "Study of phase transition and its dependence on target excitation using fractal properties of pionization process," Canadian Journal of Physics, vol. 95, no. 8, p. 699, 2017.

[47] G. Paladin and A. Vulpiani, "Anomalous scaling laws in multifractal objects," Physics Reports, vol. 156, no. 4, pp. 147-225, 1987.

[48] P. Carruthers and M. Duong-Van, "Evidence for a common fractal dimension in turbulence, galaxy distributions and hadronic multiparticle production," Los alamos preprint LA-UR-83-2419, 1983.

[49] I. M. Dermin, "The fractal correlation measure for multiple production," Modern Physics Letters A, vol. 3, no. 14, p. 1333, 1988.

[50] P. Lipa and B. Buschbeck, "From strong to weak intermittency," Physics Letters B, vol. 223, no. 3-4, pp. 465-469, 1989.

[51] H. G. E. Hentschel and I. Procaccia, "The infinite number of generalized dimensions of fractals and strange attractors," Physica D: Nonlinear Phenomena, vol. 8, no. 3, pp. 435-444, 1983.

[52] P. Grassberger and I. Procaccia, "Dimensions and entropies of strange attractors from a fluctuating dynamics approach," Physica D: Nonlinear Phenomena, vol. 13, no. 1-2, pp. 34-54, 1984.

[53] M. H. Jensen, L. P. Kadanoff, A. Libchaber, I. Procaccia, and J. Stavans, "Global universality at the onset of chaos: results of a forced rayleigh-bénard experiment," Physical Review Letters, vol. 55, no. 25, pp. 2798-2801, 1985.

[54] C. B. Chiu and R. C. Hwa, "Multifractal structure of multiparticle production in branching models," Physical Review D: Particles, Fields, Gravitation and Cosmology, vol. 43, no. 1, pp. 100-103, 1991.

[55] W. Florkowski and R. C. Hwa, "Universal multifractality in multiparticle production," Physical Review D: Particles, Fields, Gravitation and Cosmology, vol. 43, no. 5, pp. 1548-1554, 1991.

[56] M. Rybczynski, "Energy dependence of fluctuations in central $\mathrm{Pb}+\mathrm{Pb}$ collisions from NA49 at the CERN SPS," Journal of Physics G, vol. 35, Article ID 104091, 2008.

[57] E. V. Shuryak, "Quantum chromodynamics and the theory of superdense matter," Physics Reports A Review Section of Physics Letters, vol. 61, no. 2, pp. 71-158, 1980.

[58] F. Karsch, E. Laermann, and A. Peikert, "Quark mass and flavour dependence of the QCD phase transition," Nuclear Physics B, vol. 605, p. 579, 2001.

[59] F. Karsch, "Lattice results on QCD thermodynamics," Nuclear Physics A, vol. 698, no. 4, pp. 199-208, 2002.

[60] A. Bialas and W. Czyz, "Measurement of entropy of a multiparticle system: a do list," Acta Physica Polonica B, vol. 31, p. 687, 2000 . 
[61] A. Bialas and W. Czyz, "Renyi entropies in multiparticle production," Acta Physica Polonica B, vol. 31, p. 2803, 2000.

[62] A. Bialas and W. Czyz, "Renyi Entropies for Bernoulli Distributions," Acta Physica Polonica B, vol. 32, p. 2793, 2001.

[63] A. Bialas and W. Czyz, "Event by event analysis and entropy of multiparticle systems," Physical Review D: Particles, Fields, Gravitation and Cosmology, vol. 61, no. 7, 2000.

[64] P. A. Muller et al., "Quantum chaos: entropy signatures," Acta Physica Polonica B, vol. 29, p. 3643, 1998.

[65] Y. G. Ma, "Application of Information Theory in Nuclear Liquid Gas Phase Transition," Physical Review Letters, vol. 83, no. 18, pp. 3617-3620, 1999.

[66] P. Brogueira, J. Dias de Deus, and I. P. da Silva, "Information entropy and particle production in branching processes," Physical Review D: Particles, Fields, Gravitation and Cosmology, vol. 53, no. 9, pp. 5283-5285, 1996.

[67] A. Bialas, W. Czyz, and J. Wosiek, "Studying thermodynamics in heavy ion collisions," Acta Physica Polonica B, vol. 30, p. 107, 1999.

[68] A. Bialas, W. Czyz, and A. Ostruszka, "Renyi entropies in particle cascades," Acta Physica Polonica B, vol. 34, p. 69, 2003.

[69] M. Pachr, V. Simak, M. Sumbera, and I. Zborovsky, "Entropy, dimensions and other multifractal characteristics of multiplicity distributions," Modern Physics Letters A, vol. 07, no. 25, pp. 2333-2339, 1992.

[70] A. Mukhopadhyay, P. L. Jain, and G. Singh, "Entropy and fractal characteristics of multiparticle production at relativistic heavy ion interactions," Physical Review C: Nuclear Physics, vol. 47, no. 1, pp. 410-412, 1993.

[71] M. I. Adamovich, M. M. Aggarwal, and Y. A. Alexandrov, "Produced particle multiplicity dependence on centrality in nucleus-nucleus collisions," Journal of Physics G: Nuclear and Particle Physics, vol. 22, no. 10, p. 1469, 1996.

[72] M. I. Adamovich, "Erratum: stochastic emission of particles in ultra-relativistic heavy-ion collisions," Modern Physics Letters A, vol. 06, no. 17, pp. 1629-1629, 1989.

[73] F. Glück, I. Joób, and J. Lastc, "Measurable parameters of neutron decay," Nuclear Physics A, vol. 593, no. 2, pp. 125-150, 1995.

[74] M. I. Adamovich, "EMU-01 Collaboration," Physics Letters B, vol. 201, no. 1, pp. 95-100, 1988.

[75] S. Ahmad, A. Ahmad, and A. Chandra, "Forward-backward multiplicity fluctuations in $200 \mathrm{~A} \mathrm{GeV} / \mathrm{c} 16 \mathrm{O}-\mathrm{AgBr}$ and $32 \mathrm{~S}$ AgBr collisions," Physica Scripta, vol. 87, no. 4, Article ID 045201, 2013.

[76] S. Ahmad, M. M. Khan, N. Ahmad, and A. Ahmad, "Erraticity behaviour in relativistic nucleus-nucleus collisions," Journal of Physics G: Nuclear and Particle Physics, vol. 30, no. 9, pp. 11451152, 2004.

[77] S. Ahmad, M. M. Khan, S. Khan, A. Khatun, and M. Irfan, "A study of fluctuations of voids in relativistic ion-ion collisions," International Journal of Modern Physics E, vol. 24, no. 10, p. 15, 2015.

[78] M. L. Cherry et al., "KLM Collaboration," Acta Physica Polonica $B$, vol. 29, p. 2129, 1998.

[79] X. N. Wang, "A pQCD-based approach to parton production and equilibration in high-energy nuclear collisions," Physics Reports, vol. 287, no. 5-6, pp. 287-371, 1997.

[80] M. Gyulassy and X. N. Wang, "HIJING 1.0: A Monte Carlo program for parton and particle production in high energy hadronic and nuclear collisions," Computer Physics Communications, vol. 83, pp. 307-331, 1994.

[81] A. Bershadsky, "Multifractal thermodynamics of hadronhadron and hadron-nucleus interactions," The European Physical Journal A, vol. 2, p. 223, 1998.

[82] A. Arneodo, E. Bacry, and J. F. Muzy, "The thermodynamics of fractals revisited with wavelets," Physica A: Statistical Mechanics and its Applications, vol. 213, no. 1-2, pp. 232-275, 1995.

[83] E. A. De Wolf, I. M. Dremin, and W. Kittel, "Scaling laws for density correlations and fluctuations in multiparticle dynamics," Physics Reports, vol. 270, pp. 1-141, 1996.

[84] R. B. Peschanski, "Intermittency in particle collisions," International Journal of Modern Physics A, vol. 6, p. 3681, 1991.

[85] A. Bershadskii, "Multifractal specific heat," Physica A: Statistical Mechanics and its Applications, vol. 253, no. 1-4, pp. 23-37, 1998.

[86] D. Ghosh, A. Deb, P. Bandyopadhyay et al., "Evidence of multifractality and constant specific heat in hadronic collisions at high energies," Physical Review C: Nuclear Physics, vol. 65, no. 6, 2002.

[87] S. Ahmad, A. R. Khan, M. Zafar, and M. Irfan, "On multifractality and multifractal specific heat in ion-ion collisions," Chaos, Solitons \& Fractals, vol. 42, no. 1, pp. 538-547, 2009.

[88] F. Takagi, "Multifractal structure of multiplicity distributions in particle collisions at high energies," Physical Review Letters, vol. 72, no. 1, pp. 32-35, 1994.

[89] L. D. Landau and E. M. Lifshitz, Statistical Physics, Part I, Pergamon Press, Oxford, USA, 1980.

[90] D.-S. Du, X.-Q. Li, Z.-T. Wei, and B.-S. Zou, "Inelastic final-state interactions in $B \rightarrow V V \rightarrow \pi K$ processes," The European Physical Journal A, vol. 4, no. 1, pp. 91-96, 1999.

[91] Y. Ashkenazy, D. R. Baker, H. Gildor, and S. Havlin, "Nonlinearity and multifractality of climate change in the past 420,000 years," Geophysical Research Letters, vol. 30, no. 22, p. 2146, 2003.

[92] Y. U. Shimizu, S. Thurner, and K. Ehrenberger, "Multifractal spectra as a measure of complexity in human posture," Fractals, vol. 10, no. 1, pp. 103-116, 2002.

[93] G. Bhoumik, A. Deb, S. Bhattacharyya, and D. Ghosh, "Comparative multifractal detrended fluctuation analysis of heavy ion interactions at a few $\mathrm{GeV}$ to a few hundred GeV," Advances in High Energy Physics, vol. 2016, pp. 1-9, 2016.

[94] L. Telesca, "Investigating the multifractal properties of geoelectrical signals measured in southern Italy," Physics and Chemistry of the Earth, vol. 29, p. 295, 2004.

[95] J. Feder, Fractals, Plenum Press, New York, NY, USA, 1989. 

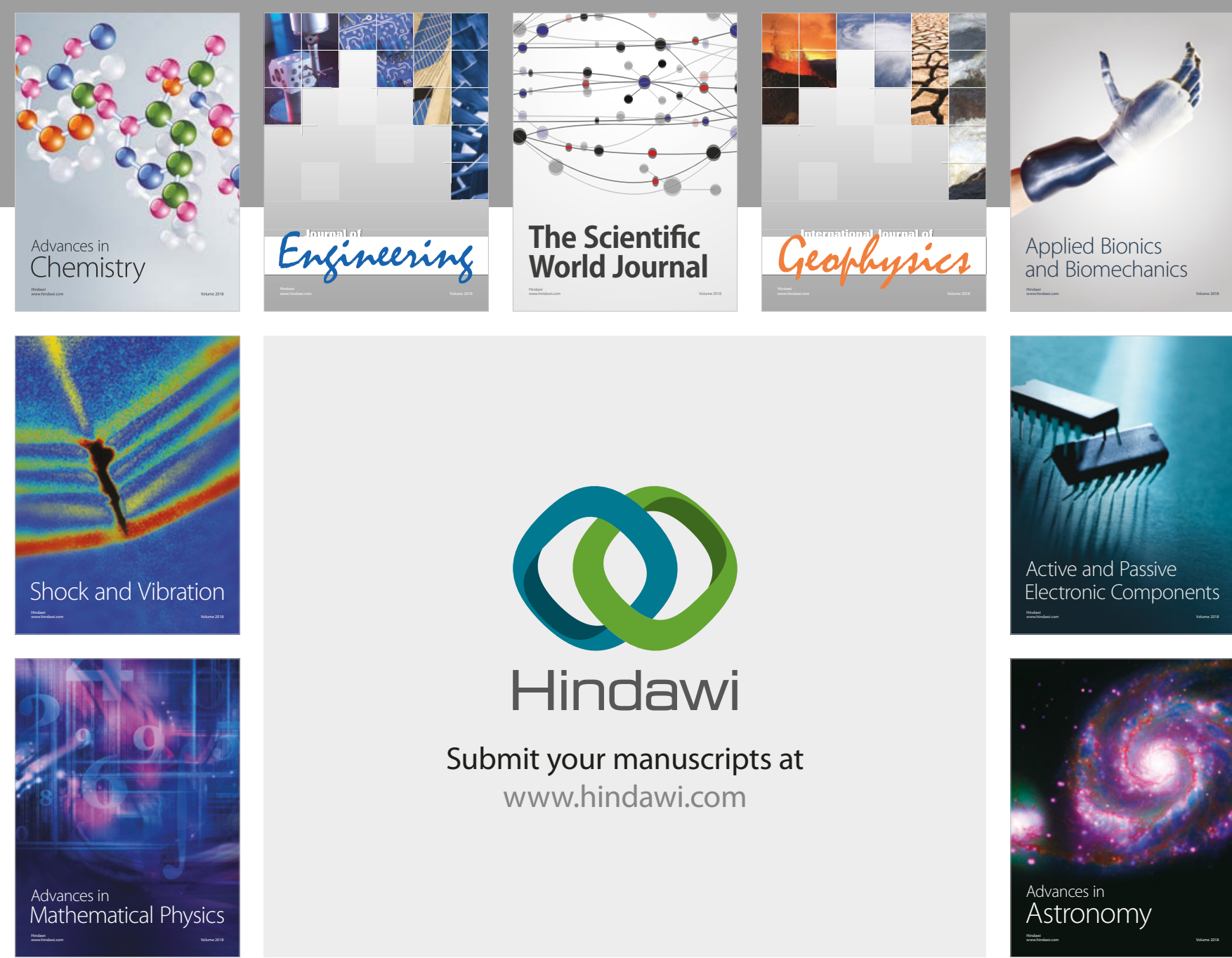

Submit your manuscripts at

www.hindawi.com

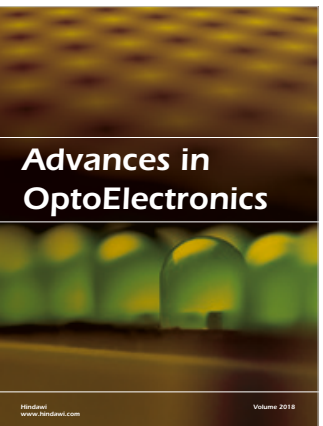

\section{Rotcting Machinery}
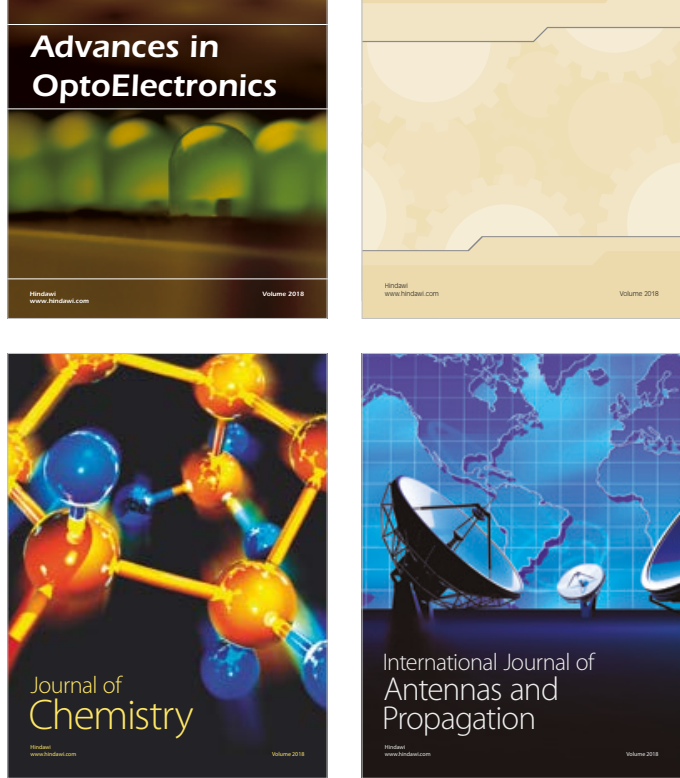

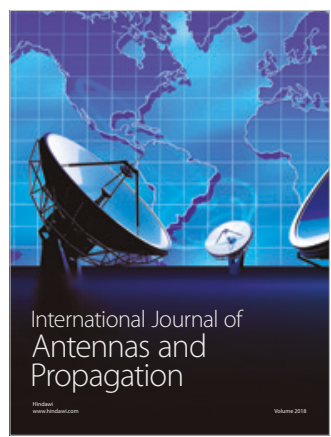

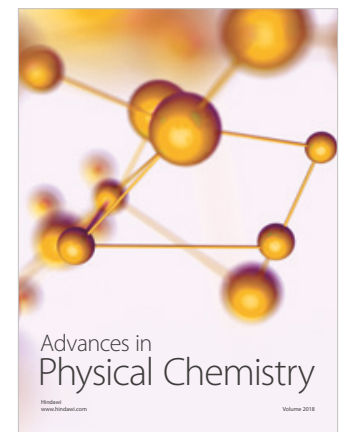

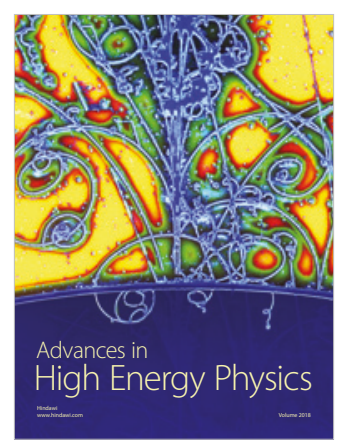

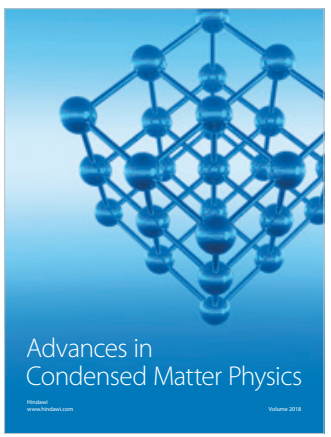

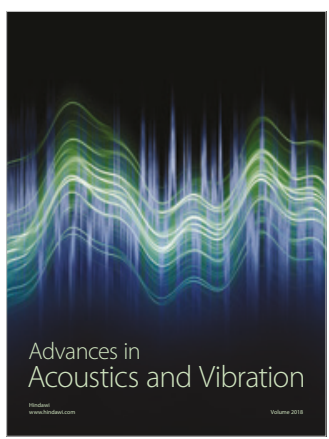

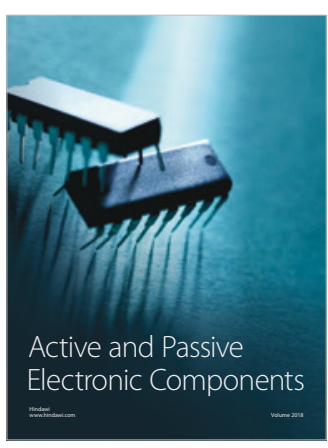
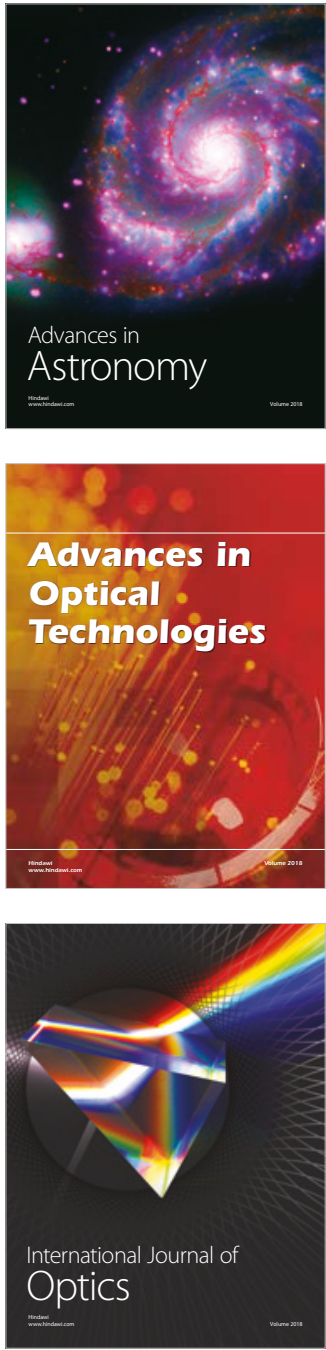